'Sección de Gastroenterología, Servicio de Medicina. Hospital San Juan de Dios. Santiago, Chile. 2 Programa Enfermedad Inflamatoria Intestinal, Departamento de Gastroenterología, Clínica Las Condes. Santiago, Chile. 3Departamento de Ginecología y Obstetricia, Departamento de Medicina Occidente. Facultad de Medicina, Universidad de Chile. Santiago, Chile. aFellow Enfermedad Inflamatoria Intestinal, Departamento Gastroenterología, Clínica Las Condes.

Los autores declaran no tener conflictos de interés.

Trabajo no recibió financiamiento.

Recibido el 22 de octubre de 2019, aceptado el 28 de mayo de 2020.

Correspondencia a: Dra. Paulina Núñez F. Huérfanos 3255, Santiago, Chile paulinanunez@gmail.com

\section{Embarazo en enfermedad inflamatoria intestinal: no solo 9 meses de cuidado}

\author{
PAULINA NÚÑEZ F. ${ }^{1, a}$, PATRICIO IBÁÑEZ L. ${ }^{2}$, GONZALO PIZARRO J. ${ }^{2}$, \\ EDUARDO SEPÚLVEDA S. ${ }^{3}$, RODRIGO QUERA P. $^{2}$
}

\section{Inflammatory bowel disease during pregnancy}

The prevalence of inflammatory bowel disease (IBD) increased in the last decades. Thus, the number of pregnant women with the condition is also increasing. Given that active disease itself is the main risk factor for complications during pregnancy, it is necessary to achieve a complete remission before planning a pregnancy. Also, pregnant women with IBD must be monitored noninvasively and be treated proactively, including escalated therapies, if needed, to prevent potential flares during pregnancy. Patients can undergo vaginal delivery in most forms of IBD. However, cesarean delivery is still preferable in women with a history of ileal pouch-anal anastomoses (IPAA) or active perianal disease.

(Rev Med Chile 2020; 148: 1806-1812)

Key words: Inflammatory Bowel Diseases; Pregnancy; Quality of Health Care.
I a enfermedad inflamatoria intestinal (EII) tiene una mayor incidencia entre los 20 y 40 años, siendo este el período reproductivo. En el embarazo, las reagudizaciones son el principal factor de complicaciones, con un aumento de eventos tromboembólicos y en lo obstétrico un aumento de abortos espontáneos, preeclampsia, necesidad de cesáreas de urgencia y recién nacidos prematuros o con bajo peso al nacer ${ }^{1-4}$.

Es importante un manejo desde la preconcepción, explicando los riesgos asociados a la actividad de la enfermedad y los beneficios de lograr y mantener remisión durante todo el embarazo ${ }^{5}$. Nos enfocaremos en la asistencia desde el periodo preconcepcional hasta el postparto.

\section{Período preconcepcional}

En edad fértil, el ginecólogo/obstetra participa activamente en las decisiones de anticoncepción. Dado que la EII aumenta 3-8 veces el riesgo de eventos tromboembólicos y que los anticon- ceptivos con estrógenos también aumentan este riesgo, estos últimos no son recomendables en EII $^{6}$. La efectividad de la terapia anticonceptiva oral disminuye si existen resecciones intestinales extensas o compromiso del intestino delgado ${ }^{7}$. Por lo anterior, los dispositivos intrauterinos implantables con liberación de progestágenos en bajas dosis debiesen ser considerados de primera línea ${ }^{8}$. Solo $25 \%$ de las pacientes con EII utiliza contracepción efectiva y $17 \%$ usa dispositivos intrauterinos?.

\section{Planificación familiar}

\section{a) Consejería preconcepcional}

La asesoría respecto a fertilidad, embarazo y lactancia es fundamental para resolver las interrogantes de las pacientes. Sobre $60 \%$ de pacientes en período fértil no quiere embarazarse por miedo a alguna complicación, ya sea por la enfermedad o por los tratamientos utilizados. Tras la consejería, el deseo de embarazo aumenta $[\mathrm{p}<0,0001]^{10}$. 
La remisión permite que los índices de fertilidad sean similares a la población general ${ }^{11,12}$, por lo tanto, para planificar un embarazo se recomienda 3-6 meses de remisión clínica (ideal con calprotectina fecal $<100$ a $200 \mathrm{ug} / \mathrm{g})^{13}$. Por el contrario, si la concepción ocurre durante una crisis, mayor es el riesgo de actividad persistente o recaídas y de complicaciones del embarazo ${ }^{14}$.

En mujeres, la proctocolectomía total con reservorio ileoanal (PCT) disminuye las tasas de fertilidad, estos valores han mejorado tras PCT laparoscópica ${ }^{15,16}$.

No existe evidencia que los fármacos utilizados en la EII reduzcan la fertilidad femenina. Por sus potenciales riesgos teratogénicos, metotrexato debe suspenderse 6 meses previo al deseo de embarazo ${ }^{17}$. Aunque existen escasos estudios con tofacitinib (moléculas pequeñas inhibidoras de Janus-kinasas), en modelos animales este fármaco se asociaría a un riesgo de malformaciones ${ }^{7}$, por lo que debiera evitarse ${ }^{18}$.

En hombres, la actividad de la enfermedad también juega un rol en la fertilidad, aquellos que están en remisión o con actividad leve no presentan diferencia con sujetos sanos ${ }^{19}$. En un estudio retrospectivo, aquellos pacientes que se encontraban activos o presentaban un brote reciente presentaron mayor dificultad para concebir (OR 2,62 IC 1,34-5,13) al compararlos con los que estaban en remisión (OR 0,93 IC $0,37-2,33)^{20}$. Sulfasalazina se asociaría a menor motilidad espermática y oligospermia, siendo este efecto reversible. Metotrexato se relacionaría a oligospermia y alteración en función reproductiva mediante acciones citotóxicas, teratogénicas y mutagénicas ${ }^{21,22}$. Aunque en hombres se ha sugerido el cambio de terapia 3-4 meses antes de concebir, se recomienda control con el gastroenterólogo para cambio de terapia, dado que los riesgos de la suspensión podrían superar a los hipotéticos beneficios $^{23}$. Aunque pocos estudios han evaluado el efecto de los anti-TNF en la ferilidad masculina, se ha señalado que infliximab no tendría asociación al desarrollo de abortos o malformaciones asociadas a la paternidad ${ }^{24}$.

El riesgo genético es una preocupación frecuente, la historia familiar es factor importante en el desarrollo de EII. La enfermedad de Crohn es la que muestra mayor asociación, con un rango entre $0,35-4,5 \%$ vs $0,3-2,7 \%$ en colitis ulcerosa ${ }^{25,26}$. Otro punto importante es conocer que el riesgo de malformaciones congénitas es similar a la población general ${ }^{27}$.

\section{b) Medidas preventivas}

Se debe enfatizar la suspensión del tabaco, lo que permitiría una mayor respuesta a terapias y a mayor éxito obstétrico ${ }^{28,29}$.

Para prevenir los defectos del tubo neural, la suplementación con ácido fólico debe iniciarse al menos un mes antes del deseo de concepción ${ }^{30}$. Si existe compromiso de intestino delgado o resección extensa la dosis debe ser $5 \mathrm{mg} /$ día.

En embarazadas, se recomienda la inmunización contra influenza desde la semana 13 del embarazo. Los beneficios se extienden al recién nacido, dado que los anticuerpos traspasan la placenta $^{31}$.

\section{Embarazo}

La actividad de la enfermedad se asocia a bajo peso al nacimiento, bajos puntaje de test de Apgar e hipoglicemia ${ }^{32}$. Si no aumenta de peso adecuadamente, es mayor el riesgo de hijos pequeños para la edad gestacional ${ }^{33}$.

\section{a) Monitorización de la actividad inflamatoria durante el embarazo}

Los síntomas durante las crisis son similares que en población $\mathrm{EII}^{12}$. Se deben solicitar hemograma, proteína $\mathrm{C}$ reactiva y calprotectina fecal. Este último determina la presencia de actividad inflamatoria con un valor predictivo positivo de $66,7-74,4 \%{ }^{34}$.

Se debe realizar colonoscopía solo si existe un beneficio real como, por ejemplo, para optimizar terapia $^{35}$. La colonoscopia sería de bajo riesgo durante todo el embarazo, aunque se debe considerar los riesgos de los sedantes, esta puede realizarse sin preparación y sin sedación ${ }^{36}$.

\section{b) Medicamentos en embarazo y lactancia (Figura 1)}

\section{1) 5-aminosalicilatos}

El componente ftalato de dibutilo o dibutilftalato en la cubierta de algunos formas farmacéuticas de algunos derivados 5-ASA (Asacol ${ }^{\circledR}$ y Mecolzine ${ }^{\circledR}$ ), se ha asociado a un incremento de malformaciones urinarias en modelos de anima- 
Pre concepción

Metotrexato suspender 6 meses previo a embarazo.

Talidomida

5 ASA con ftalato de dibutilo: Asacol $®$ Mecolzine ${ }^{\circledR}$

Tofacitinib : suspender 1 semana previo a deseo de embarazo

Insuficientes datos:

Anti-integrinas

anti-interleukinas 12/23

\section{5- ASA sin ftalato de dibutilo}

Sulfasalazina agregar ácido fólico

Tiopurinas (Aza y $6 \mathrm{MP}$ )

Anti TNF $\alpha$ : IFX , ADA , golimumab

certolizumab

\section{Embarazo}

Metotrexato (teratogénico)

Talidomida

No usar 5-ASA con ftalato de dibutilo

Tofacitinib (al menos en el 1er

trimestre del embarazo)

\section{Insuficientes datos:}

Anti-integrinas

anti-interleukinas 12/23

\section{5- ASA sin ftalato de dibutilo} Sulfasalazina agregar ácido fólico)

Tiopurinas (Aza y $6 \mathrm{MP}$ )

Anti TNF $\alpha$ : IFX ADA y golimumab

suspender finales del 3er trimestre

según semanas de gestación.

Certolizumab: mantener

\section{La actividad de la enfermedad es el mayor riesgo, No se deben suspender terapias.}

5-ASA: 5-aminosalicilatos; Aza :azatioprina; 6-MP: 6-mercaptopurina; Anti TNF $\alpha$ : anti factor necrosis tumoral $\alpha$; IFX: infliximab; ADA: adalimumab

Figura 1. Fármacos en pacientes con Ell en edad fértil.

les ${ }^{37}$. Si bien, estos resultados no han sido descritos en humanos ${ }^{12}$, parece prudente evitarlos ${ }^{38}$.

Sulfasalazina y su metabolito sulfapiridina cruzan la placenta y pueden inhibir la síntesis de folato, siendo necesario suplementarlo.

\section{2) Corticoides}

Los corticoides (excepto prednisona) cruzan la placenta, sin embargo, el riesgo de malformaciones orofaciales durante el primer trimestre no ha sido confirmado ${ }^{39}$. Deben utilizarse solo en crisis $^{5}$, considerando siempre los riesgos maternos como hipertensión arterial, diabetes mellitus y preeclampsia $^{12}$. Budesonida MMX podría ser útil en reactivación ${ }^{40}$.

\section{3) Inmunomoduladores}

En el registro PIANO ${ }^{41}$, azatioprina no se asoció con anomalías congénitas ni complicaciones del embarazo. Sí hubo riesgo de infecciones en niños expuestos hasta los 9-12 meses, siendo ma- yor en terapia combinada (con anti-TNF) (RR1,5 IC 95\% 1,98-2,09) $)^{42}$.

Parece razonable continuar con monoterapia durante el embarazo para mantener la remisión, sin embargo, nunca debe ser iniciada en el embarazo dado su lenta acción y los eventos adversos asociados ${ }^{43}$. Metotrexato y talidomida están contraindicados por sus efectos adversos ${ }^{44}$.

\section{4) Anti-TNF}

Los anti-TNF no presentan mayores tasas de efectos adversos durante el embarazo ${ }^{12,45,46}$. Estos biológicos cruzan la placenta en el $2^{\circ}$ y $3^{\text {er }}$ trimestre uniéndose a la fracción constante (FC) IgG1 presentes en las células placentarias (sinciotrofoblastos $)^{46}$. La excepción es certolizumab que por su porción pegilada, no atraviesa la placenta ${ }^{47,48}$. Se han detectado niveles plasmáticos de anti-TNF en concentraciones mayores a las maternas hasta 6 meses de vida, por lo que deben evitarse las vacunas vivas ${ }^{49}$. 


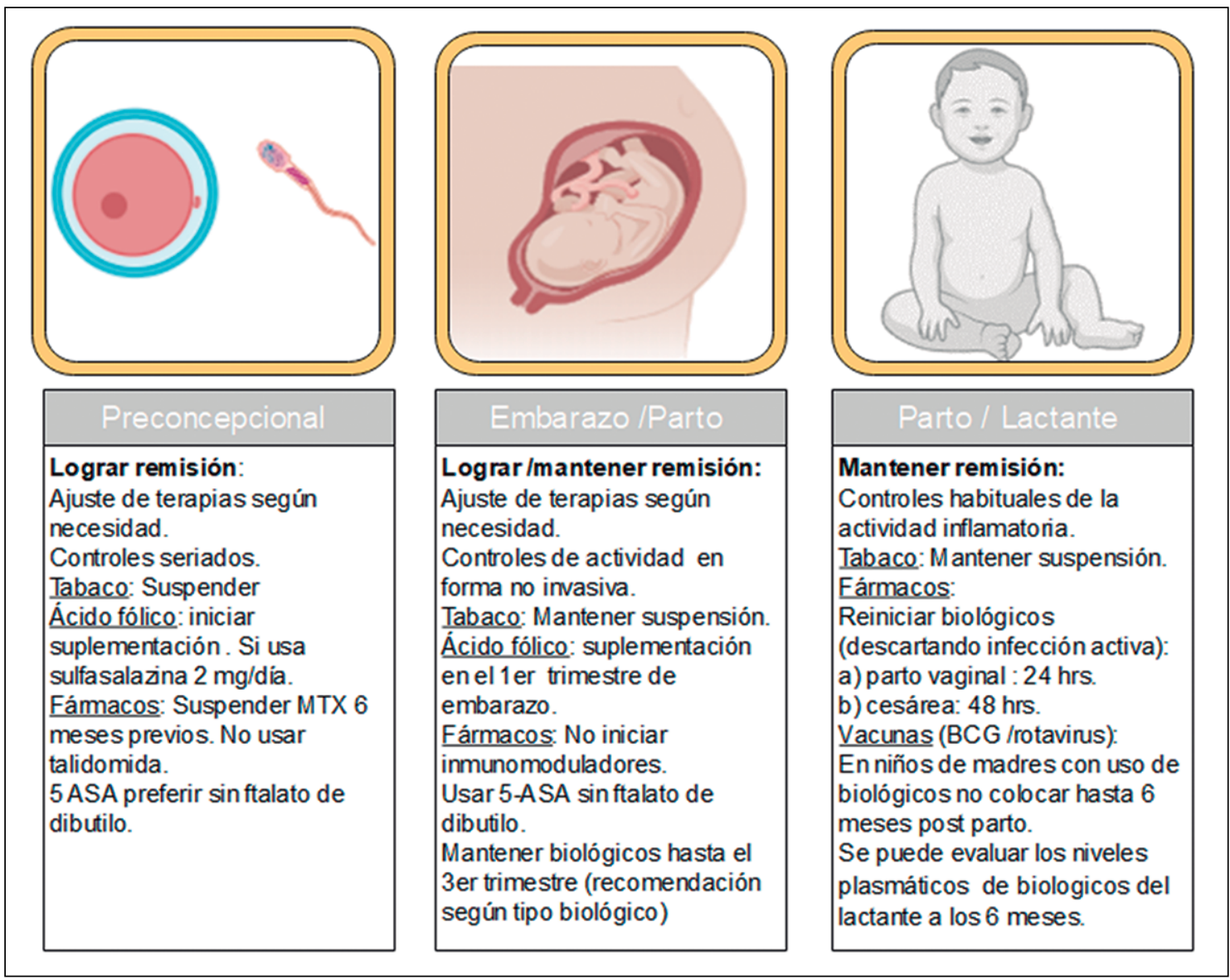

Figura 2. Cuidados en pre concepción/embarazo y lactancia. MTX: metotrexato; 5-ASA: 5-amino salicílicos; BCG: bacillus Calmetté-Guérin (tuberculosis).

Si bien no hay evidencia de que continuar con anti-TNF durante el embarazo tenga un impacto negativo en el embarazo y parto, en aquellas con enfermedad activa durante el segundo semestre, el beneficio de continuar la terapia anti-TNF durante el tercer trimestre superaría el riesgo potencial. Sin embargo, para evitar el traspaso placentario aún se recomienda que la última dosis sea: infliximab 30-32 semanas, adalimumab 36-38 y golimumab 34-36 semanas ${ }^{50,51}$.

Un seguimiento a 4 años no evidenció que el riesgo de infección grave en los recién nacidos se relacione con niveles plasmáticos del fármaco en ellos ${ }^{52,53}$. Adalimumab tampoco reporta riesgos en el embarazo o en el recién nacido ${ }^{54}$. Las infecciones y embarazos pretérminos serían mayor en terapia combinada (OR 2,4; IC 95\% 1,3-4,3) $)^{45}$.

\section{5) Antiintegrinas y antiinterleukinas $12 / 23$}

Hasta ahora existen estudios limitados, no es posible una recomendación ${ }^{55-57}$.

\section{c) Programación del parto}

La mayoría de las mujeres con EII pueden tener un parto vaginal, pero dependerá de las necesidades obstétricas ${ }^{7}$. Sin embargo, la cesárea debe sugerirse en enfermedad perianal activa e historia de $\mathrm{PCT}^{58}$.

Los medicamentos biológicos deben reiniciarse $24 \mathrm{~h}$ postparto vaginal o $48 \mathrm{~h}$ tras cesárea. En el recién nacido es perentorio la suspensión de vacuna a virus vivos por al menos 6 meses, en caso de dudas, se recomienda tomar niveles de anti-TNF en el lactante ${ }^{45,59}$. 


\section{Cuidados postparto}

El postparto se convierte en un escenario ideal de prevención. Estudios indican que el porcentaje de pesquisa de cáncer cervicouterino postparto es mayor $84 \%$ vs $70,4 \%$ del total de mujeres EII $^{60}$.

Dado que el embarazo y la lactancia son períodos donde puede disminuir la densidad ósea, siendo crítico en mujeres con historia de uso de corticoides, los cuidados deben enfocarse en el metabolismo óseo ${ }^{61}$.

\section{Conclusión}

La mayor incidencia de la EII se observa en el período de mayor fertilidad (o periodo fértil). El embarazo es una condición que genera preocupación, dado los riesgos propios de la enfermedad y los fármacos que se utilizan. El factor más importante para el éxito del embarazo es el control de la enfermedad, siendo la sugerencia continuar con la terapia durante el embarazo con el fin de mantener la remisión. La asesoría preconcepcional y los controles seriados con el gastroenterólogo y ginecobstetra son vitales para lograr estos objetivos (Figura 2). Se debe monitorizar la enfermedad de forma no invasiva y disponer de terapias que permitan controlar las crisis de la enfermedad.

\section{Referencias}

1. Wieringa J, Driessen G, van der Woude J. Pregnant women with inflammatory bowel disease: the effects of biologicals on pregnancy, outcome of infants and the developing immune system. Expert Rev Gastroenterol Hepatol 2018; 12 (8): 811-8.

2. Norgard M, Jolving LR, Larsen MD, Friedman S. Parenteral IBD and long term health outcomes in the offspring. Inflamm Bowel Dis 2019; 25 (8): 1339-48.

3. Shand AW, Chen JS, Selby W, Solomon M, Roberts CL. Inflammatory bowel disease in pregnancy: a population-based study of prevalence and pregnancy outcomes. BJOG 2016; 123: 1862-70.

4. Gomes CF, Sousa M, Lourenço I, Martins D, Torres J. Gastrointestinal diseases during pregnancy: what does the gastroenterologist need to know? Ann Gastroenterol 2018; 31 (4): 385-94.

5. Bell SJ, Flanagan EK. Updates in the management of inflammatory bowel disease during pregnancy. Med J Aust 2019: 210: 276-80.

6. Limdi JK, Farraye J, Cannon R, Woodhams E, Farraye FA. Contraception, venous Thromboembolism, and inflammatory bowel disease: what clinicians (and patients) should know. Inflamm Bowel Dis. 2019;25:1603-12.

7. Mahadevan U, Robinson C, Bernasko N, Boland B, Chambers C, Dubinsky M, et al. Inflammatory bowel disease in pregnancy clinical care pathway: A report from the American Gastroenterological Association IBD parenthood project working group. Inflamm Bowel Dis 2019; 25: 627-41.

8. Gawron LM, Sanders J, Steele KP, Flynn AS. Reproductive planning and contraception for women with inflammatory bowel disease. Inflamm bowel dis 2016; 22: 459-64.

9. Gawron LM, Gawron AJ, Kasper A, Hammond C, Keefer L. Contraceptive method selection by women with inflammatory bowel disease: a cross- sectional survey. Contraception. 2014; 89: 419-25.

10. Ellul P, Zammita SC, KAtsanos KH, Cesarini M, Alocca M, Danese S, KAratzas P. Perception of reproductive health in women with inflammatory bowel disease. J Crohns Colitis 2016; 10: 886-91.

11. Bell S, Flanagan E. Updates I the management of inflammatory bowel disease during pregnancy. J Aust 2019; 210: 276-80.

12. Magro F, Gionchetti P, Eliakim R, Ardizzone S, Armuzzi A, Barreiro-de Acosta M, et al. Third European evidence based consensus on diagnosis and management of ulcerative colitis. Art 1: definitions, diagnosis, extra- intestinal manifestations, pregnancy, cancer surveillance, surgery, and ileo-anal pouch disorders. J Crohns Colitis 2017; 11: 649-70.

13. Ungaro R, Colombel JF, Lissos T, Peyrin-Biroulet L. A treat-to- target Update in Ulcerative Colitis: A Systematic Review. Am J Gastroenterol. 2019; 114: 874-83.

14. Abhyankar A, Ham M, Moss AC. Meta-analysis: the impacto $\mathrm{f}$ disease activity at conception on disease activity during pregnancy in patients with inflammatory bowel disease. Aliment Pharmacol Ther 2013; 38: 460-6.

15. Beyer-Berjot L, Maggiori L, Birnbaum D, et al. A total laparoscopic approach reduces the infertility rate after ileal pouch-anal anastomosis: A 2-center study. Ann Surg 2013; 258 (2): 275-82.

16. Oza SS, Pabby V, Dodge LE, Hacker MR, Fox JH, Moragianni VA, et al. Factors associated with the success of in vitro fertilization in women with inflammatory bowel disease. Dig Dis Sci 2016.

17. Kanis SL, van der Woude. Roper use of inflammatory bowel disease. Dig Dis 2016; 34: 61-6. 
18. Dowty ME, Lin J, Ryder TF, Wang W, Walker GS, Vaz A, et al. The pharmacokinetics, metabolism, and clearance mechanisms of tofacitinib, a janus kinase inhibitor, in humans. Drug Metab Dispos 2014; 42: 759-73.

19. Timmer A, Bauer A, Dignass A, Rogler G. Sexual function in persons with inflammatory bowel disease: a survey with matched controls. Clin Gastroenterol Hepatol 2007; 5: 87-94.

20. Ananthakrishnan AN, Martin C, Kane S, Sandler RS, Long MD. Paternal disease activity is associated with difficulty in conception among men with inflammatory bowel diseases. Clin Gastroenterol Hepatol 2019;17:2034.

21. Sands K, Jansen R, Zaslau S, Greenwald D. Review article: the safety of therapeutic drugs in male inflammatory bowel disease patients wishing to conceive. Aliment Pharmacol Ther 2015; 41: 821-34.

22. Ley D, Jones J ,Parris J, Salih S, CalderaF, Tirado E et al. Methotrexate reduces DNA integrity in sperm from men with inflammatory bowel disease. Gastroenterology 2018; 154: 2064-7.

23. Palomba S, Sereni G, Falbo A, Beltrami M, Lombardini $\mathrm{S}$, Boni $\mathrm{M}$, et al. Inflammatory bowel diseases and human reproduction: a comprehensive evidence-based review. World J Gastroenterol 2014; 20: 7123-36.

24. Puchner R, Danninger K, Puchner A, Pieringer H. Impact of TNF-blocking agents on male sperm characteristics and pregnancy outcomes in fathers exposed to TNF-blocking agents at time of conception. Clin Exp Rheumatol 2012; 30: 765-7.

25. Santos MPC, Gomes C, Torres J. Familial and ethnic risk in inflammatory bowel disea Ann Gastroenterol 2018; 31: 14-23.

26. Moller FT, Andersen V, Wohlfahrt J, Jess T. Familial risk of inflammatory bowel disease: a population-based cohort study 1977-2011. Am J Gastroenterol 2015; 110: 564-71.

27. Mahadevan U, Sandborn W, Li DK, Hakimian S, Kane $\mathrm{S}$, Corley DA, et al. Pregnancy outcomes in womwn with inflammatory bowel disease: a large community -based study from Northern California. Gastroenterology 2007; 133: 1106-12.

28. Burke KE, Boumitri C, Ananthakrishnan AN. Modifiable Environmental factors in inflammatory bowel disease.Curr Gastroenterol Rep 2017; 19: 21.

29. Seirafi M, de Vroey B, Amiot A, Seksik P, Roblin X, Allez $\mathrm{M}$, et al. Factors associated with pregnancy outcome in anti- TNF treated women with inflammatory bowel disease. Aliment Pharmacol Ther 2014; 40: 363-73.

30. Van der Woude CJ, Ardizzone S, Bengtson MB, Fiorino G, Fraser G, Katsanos K, et al. The second European evi- denced based consensus on reproduction and pregnancy in inflammatory bowel disease. J Crohns Colitis 2015; 9: 107-24.

31. Zerbo O, Modaressi S, Chan B, Goddard K, Lewis N, Bok K, et al. No association between influenza vaccination during pregnancy and adverse birth outcomes. Vacinne 2017; 31: 3186-90.

32. Bröms G, Granath F, Linder M, Stephansson O, Elmberg M, Kieler H. Birth outcomes in women with inflammatory bowel disease: effects of disease activity and drug exposure. Inflamm Bowel Dis 2014; 20: 1091-8.

33. Bengtson MB, Aamodt G, Mahadevan U, Vatn MH. Inadequate gestational weight gain, the hidden link between maternal IBD and adverse pregnancy outcomes: results from the Norwegian mother and child cohort study. Inflamm Bowel Dis 2017; 23: 1225-33.

34. Kammerlander H, Nielsen J, Kjeldsen J, Knudsen T, Gradel KO, Friedman S, et al. Fecal calprotectin during pregnancy in women with moderate-severe inflammatory bowel disease. Inflamm Bowel Dis 2018; 24: 839-48.

35. Choden T, MAndaliya R, Charbaty A, Mattar M. Monitoring inflammatory bowel disease during pregnancy: Current literature and future challenges. World J Gastrointest Pharmacol Ther 2018; 9: 1-7

36. De Lima A, Galjart B, Wisse PH, Bramer WM, van der Woude CJ. Does lower gastrointestinal endoscopy during pregnancy pose a risk for mother and child? A systematic review. BMC Gastroenterol 2015; 12: 475-9.

37. Hernandez-Diaz S, Su Y-C, Mitchell AA, Kelley KE, Calafat AM, Hauser R. Medications as a potential source of exposure to phthalates among women of childbearing age. Reprod Toxicol 2013; 37: 1-5.

38. Ban L, Tata LJ, Fiaschi L, Card T. Limited risk of major congenital anomalies in children of mothers with IBD and effects of medications. Gastroenterology 2014; 146: 76-84.

39. Hviid A, Molgaard-Nielsen D. Corticosteroid use during pregnancy and risk of orofacial clefts. CMAJ 2011; 183: 796-804.

40. Vestergaard T, Jorgensen SMD, Christensen LA, Julsgaard M. Pregnancy outcome in four women with inflammatory bowel disease treated with budesonide MMX. Scand J Gastroenterol 2018; 53: 1459-62.

41. Mahadevan U, Martin C, Sandler R, Kane S, Dubinsky M, Lewis J, et al. 865 PIANO: a 1000 patient prospective registry of pregnancy outcomes in women with IBD exposed to immunomodulators and biologic therapy. Gastroenterology 2012; 142: S-149.

42. Kanis SL, de Lima-Karagiannis A, de Boer NK, van der Woude CJ. Use of thiopurines during conception and 
pregnancy is not associated with adverse pregnancy outcomes or health of infants at one year in a prospective study. Clin Gastroenterol Hepatol 2017; 15: 1232-41.

43. Mahadevan U, Mc Connell RA, Chambers CD. Drugs safety and risk of adverse outcomes for pregnant patients with inflammatory bowel disease. Gastroenterology 2017; 152: 451-62.

44. Maliszewska A, Warska A, Cendrowwski K, Sawicki W. Inflammatory bowel disease and pregnancy. Ginekol Pol 2017; 88: 398-403.

45. Shihab Z, Yeomans ND, De Cruz P. Anti-tumour necrosis factor alpha therapies and inflammatory bowel disease pregnancy outcomes: a meta-analysis. J Crohns Colitis 2016; 10: 979-88.

46. Mahadevan U, Wolf DC, Dubinsky M, Cortot A, Lee $\mathrm{SD}$, Siegel CA, et al. Placental transfer of anti-tumor necrosis factor agents in pregnant patients with inflammatory bowel disease. Clin Gastroenterol Hepatol 2013; 11: 286-92.

47. Porter C, Armstrong -Fisher S, Kopotsha T, Smith B, Baker T, Kevorkian L. Certolizumab pegol does not bind the neonatal Fc receptor: Consequences for FcRn- mediated in vitro transcytosis and ex vivo human placental transfer. J Reprod Inmunol 2016; 116: 7-12.

48. Mariette X, Förger F, Abraham B, Flynn AD, Moltó A, Flipo RM et al. Lack of placental transfer of certolizumab pegol during pregnancy: results from CRIB, a prospective, postmarketing, pharmacokinetic study. Ann Rheum Dis 2018; 77: 228-33.

49. Julsgaard M, Christensen LA, Gibsn PR, Gearry RB, Fallingborg J, Hvas CL, et al. Concentrations of Adalimumab and Infliximab in mothers and newborns, and effects on infection. Gastroenterology 2016; 151: $110-9$.

50. Zelinkova Z, van der Ent C, Bruin K, van Baalen O, Vermeulen $\mathrm{H}$, Smalbraak H, et al. Effects of discontinuing anti-tumor necrosis factor therapy during pregnancy on the course of Inflammatory bowel disease and neonatal exposure. Clin Gastroenterol Hepatol 2013; 11: 318-21.

51. Mc Connell R, Mahadevan U. Pregnancy and the patient with inflammatory bowel disease. Gastroenterol Clin
North Am 2016; 45: 285-301.

52. Chaparro M, Verreth A, Lobaton T, Gravito-Soares E, Julsgaard M, Savarino E, et al. Longterm safety of in útero exposure to Anti- TNF $\alpha$ drugs for the treatment of inflammatory bowel disease: results from the multicenter European TEDDY study. Am J Gastroenterol 2018; 113: 396-403.

53. Duricova D, Dvorakova E, Hradsky O,Mitrova K, Durilova M, Kozeluhova J. Safety of Anti TNF Alpha therapy during pregnancy on long term outcome of exposed children: A controlled, multicenter observation. Inflamm Bowel Dis 2019; 14: 789-96.

54. Kawai Y, Tsuchiya T, Aoki S. Pregnany outcomes of patients exposed to adalimumab in Japan. Dig Dis 2019; 37: 123-30.

55. Peng A, Qiu X, Zhang L, Zhu X, He S, Lai W, et al. Natalizuab exposure during pregnancy in multiple sclerosis: a systematic review. J Neurol Sci 2019; 396: 202-5.

56. Madadevan U, Vermeire S, LAsch K, Abhyankar B, Bhayat $\mathrm{F}$, lake A, et al. Vedolizumab exposure in pregnancy: outcomes from clinical studies in inflammatory bowel disease. Aliment Pharmacol Ther 2017; 45: 941-50.

57. Deepak P, Sandborn WJ. Ustekinumab and anti-interleukin- 23 agents in Crohn's disease. Gastroenterol Clin North Am 2017; 46: 603-26.

58. Burke K, Haviland M, Hacker M, Shainker S, Cheifetz A. Indications for mode of delivery in pregnant women with inflammatory bowel disease.

59. Restellini S, Biedermann L, Hruz P, Mottet C, Moens A, et al. Update on the management of inflammatory bowel disease during pregnancy and breastfeeding. Digestion 2020; 101 Suppl 1: 27-42.

60. Mao EJ, Sheibani S, Martin C, Bloomfeld RS, Isaacs K, Saha $S$, et al. Preventive health care among post partum women with inflammatory bowel disease: Results from the PIANO registry. Inflamm Bowel Dis 2019; 25: 797802.

61. Farraye FA; Melmed GY, Lichtenstein GR, Kane SV. AACG clinical guideline: Preventive care in Inflammatory bowel disease. Am J Gastroenterol 2017; 112: 241-58. 